\title{
Prairie Bird Species of Concern - Ecology and Management Summary
}

Each grassland bird species requires unique prairie conditions. Subtle differences across the landscape determine the suitability of local habitat for different species for different reasons.

\begin{tabular}{|c|c|c|c|c|c|c|c|c|c|c|}
\hline $\begin{array}{l}\text { Species Name } \\
\text { Species Status }\end{array}$ & $\begin{array}{l}\text { General Habitat } \\
\text { Characteristics }\end{array}$ & $\begin{array}{c}\text { Nest Site } \\
\text { Characteristics }\end{array}$ & $\begin{array}{c}\text { Management } \\
\text { Recommendations to } \\
\text { Promote Species }\end{array}$ & Notes & $\begin{array}{c}\begin{array}{c}\text { Arrival } \\
\text { Date }\end{array} \\
\begin{array}{c}\text { Departure } \\
\text { Date }\end{array} \\
\end{array}$ & $\begin{array}{l}\text { Nesting } \\
\text { Dates }\end{array}$ & $\begin{array}{c}\text { Incubation } \\
\text { Period } \\
\text { Days to } \\
\text { Fledging } \\
\end{array}$ & $\begin{array}{l}\text { Number } \\
\text { of } \\
\text { Broods }\end{array}$ & $\begin{array}{c}\text { Primary } \\
\text { Summer Diet }\end{array}$ & $\begin{array}{l}\text { Secondary } \\
\text { Summer } \\
\text { Diet }\end{array}$ \\
\hline \multicolumn{11}{|c|}{ Low to Intermediate Grassland Cover Associated Species } \\
\hline $\begin{array}{l}\text { McCown's } \\
\text { Longspur }^{1} \\
\text { prairie endemic } \\
\text { G5/S2B }\end{array}$ & $\begin{array}{l}\text { Open sparse } \\
\text { grassland; areas } \\
\text { structurally } \\
\text { similar to heavily } \\
\text { grazed } \\
\text { pastures. }\end{array}$ & $\begin{array}{l}\text { Ground nester. } \\
\text { Shallow } \\
\text { depression in the } \\
\text { ground: either in } \\
\text { the open or } \\
\text { beside vegetation. }\end{array}$ & $\begin{array}{l}\text { Maintain short, sparsely } \\
\text { vegetated native prairie. } \\
\text { Moderate to heavy, or } \\
\text { season long grazing can } \\
\text { make mixed-grass prairie } \\
\text { suitable. }\end{array}$ & $\begin{array}{l}\text { Limited shrubs in } \\
\text { area are } \\
\text { suitable; } \\
\text { Nestlings suffer } \\
\text { high rates of } \\
\text { (mammalian) } \\
\text { predation. }\end{array}$ & $\begin{array}{l}\text { Late April } \\
\text { Mid-August }\end{array}$ & $\begin{array}{l}\text { Early May } \\
\text { to Late } \\
\text { July }\end{array}$ & 10-12 days & 2 & $\begin{array}{l}\text { seeds/plant } \\
\text { material }\end{array}$ & insects \\
\hline $\begin{array}{l}\text { Chestnut- } \\
\text { collared } \\
\text { Longspur }{ }^{2} \\
\text { prairie endemic } \\
\text { G5/S3B }\end{array}$ & $\begin{array}{l}\text { Sparse; recently } \\
\text { grazed, mowed, } \\
\text { or burned; } \\
\text { minimal litter. }\end{array}$ & $\begin{array}{l}\text { Ground nester: } \\
\text { often protected on } \\
\text { south and east } \\
\text { sides }\end{array}$ & $\begin{array}{l}\text { Keep native pastures } \\
\text { intact. Manage for fairly } \\
\text { short vegetation with } \\
\text { sparse litter accumulation. }\end{array}$ & $\begin{array}{l}\text { Native prairie } \\
\text { specialist. } \\
\text { Areas with } \\
\text { occasional } \\
\text { shrubs suitable. }\end{array}$ & $\begin{array}{l}\text { Mid-April } \\
\text { Late } \\
\text { September }\end{array}$ & $\begin{array}{l}\text { Early May } \\
\text { to Late } \\
\text { July }\end{array}$ & 10-13 days & 2 & insects & seeds \\
\hline $\begin{array}{l}\text { Lark Bunting }^{3} \\
\text { prairie endemic } \\
\text { G5/S3B }\end{array}$ & $\begin{array}{l}\text { Primarily short- } \\
\text { to-mid grass } \\
\text { prairie. } \\
\text { Prefers areas } \\
\text { with some } \\
\text { shrubs. } \\
\text { Generally } \\
\text { prefers areas } \geq \\
10 \mathrm{~km}^{2} \text {. }\end{array}$ & $\begin{array}{l}\text { Ground nester. } \\
\text { Nest placed next } \\
\text { to/under various } \\
\text { plant species }\end{array}$ & $\begin{array}{l}\text { Provide areas of short } \\
\text { vegetation with protective } \\
\text { cover for nesting. }\end{array}$ & $\begin{array}{l}\text { May be present } \\
\text { in agricultural } \\
\text { fields, but use is } \\
\text { limited. Highest } \\
\text { densities in } \\
\text { native prairie, } \\
\text { though will nest } \\
\text { in CRP lands. }\end{array}$ & $\begin{array}{l}\text { Early May } \\
\text { Late } \\
\text { August }\end{array}$ & $\begin{array}{l}\text { Mid-May } \\
\text { to Mid- } \\
\text { August }\end{array}$ & 8-9 days & 1 to 2 & insects & $\begin{array}{l}\text { secondary } \\
\text { seeds/plant } \\
\text { material }\end{array}$ \\
\hline $\begin{array}{l}\text { Long-billed } \\
\text { Curlew }{ }^{4} \\
\text { prairie endemic } \\
\text { G5/S2B }\end{array}$ & $\begin{array}{l}\text { Open sparse } \\
\text { grassland } \\
\text { preferred; taller, } \\
\text { denser grass } \\
\text { during brood } \\
\text { rearing. }\end{array}$ & $\begin{array}{l}\text { Ground nester. } \\
\text { Nest is a scrape } \\
\text { in the ground } \\
\text { lined with various } \\
\text { bits of vegetation, } \\
\text { pebbles, and } \\
\text { other organic } \\
\text { matter. }\end{array}$ & $\begin{array}{l}\text { Provide large, open level to } \\
\text { gently rolling grasslands } \\
\text { with short vegetation. }\end{array}$ & $\begin{array}{l}\text { Generally avoids } \\
\text { areas with high } \\
\text { density of } \\
\text { shrubs. }\end{array}$ & $\begin{array}{l}\text { Early April } \\
\\
\text { Mid- } \\
\text { September }\end{array}$ & $\begin{array}{l}\text { Mid-to } \\
\text { Late April }\end{array}$ & $\begin{array}{l}28-31 \text { days } \\
\text { (able to } \\
\text { leave nest } \\
\text { within hours } \\
\text { of hatching) } \\
32-45 \text { days }\end{array}$ & 1 & $\begin{array}{l}\text { carnivorous } \\
\text { (terrestrial } \\
\text { invertebrates, } \\
\text { vertebrates, } \\
\text { eggs, etc.) }\end{array}$ & - \\
\hline
\end{tabular}




\begin{tabular}{|c|c|c|c|c|c|c|c|c|c|c|}
\hline $\begin{array}{l}\text { Species Name } \\
\text { Species Status }\end{array}$ & $\begin{array}{l}\text { General Habitat } \\
\text { Characteristics }\end{array}$ & $\begin{array}{c}\text { Nest Site } \\
\text { Characteristics }\end{array}$ & $\begin{array}{l}\text { Management } \\
\text { Recommendations to } \\
\text { Promote Species }\end{array}$ & Notes & $\begin{array}{c}\begin{array}{c}\text { Arrival } \\
\text { Date }\end{array} \\
\begin{array}{c}\text { Departure } \\
\text { Date }\end{array} \\
\end{array}$ & $\begin{array}{l}\text { Nesting } \\
\text { Dates }\end{array}$ & $\begin{array}{c}\begin{array}{c}\text { Incubation } \\
\text { Period }\end{array} \\
\text { Days to } \\
\text { Fledging } \\
\end{array}$ & $\begin{array}{l}\text { Number } \\
\text { of } \\
\text { Broods }\end{array}$ & $\begin{array}{c}\text { Primary } \\
\text { Summer Diet }\end{array}$ & $\begin{array}{l}\text { Secondary } \\
\text { Summer } \\
\text { Diet }\end{array}$ \\
\hline $\begin{array}{l}\text { Ferruginous } \\
\text { Hawk } \\
\text { prairie endemic } \\
\text { G4/S2B }\end{array}$ & $\begin{array}{l}\text { Prefer open } \\
\text { prairie and } \\
\text { shrubsteppe } \\
\text { habitat. }\end{array}$ & $\begin{array}{l}\text { Exposed: on cliffs, } \\
\text { trees, ground, or } \\
\text { manmade } \\
\text { structures. }\end{array}$ & $\begin{array}{l}\text { Provide/protect suitable } \\
\text { nest sites, protect active } \\
\text { nest areas from } \\
\text { disturbance, and improve } \\
\text { habitat for prey. }\end{array}$ & $\begin{array}{l}\text { Prefers flat, } \\
\text { rolling terrain. } \\
\text { Avoids high } \\
\text { elevation, } \\
\text { forested areas } \\
\text { and narrow } \\
\text { canyons. Avoids } \\
\text { areas recently } \\
\text { altered for } \\
\text { cultivation }\end{array}$ & $\begin{array}{c}\text { March } \\
\text { Late } \\
\text { September }\end{array}$ & $\begin{array}{l}\text { Late April } \\
\text { to Mid- } \\
\text { July }\end{array}$ & 44-48 days & 1 & $\begin{array}{c}\text { small } \\
\text { mammals (inc. } \\
\text { jackrabbits, } \\
\text { cottontail } \\
\text { rabbits, } \\
\text { ground } \\
\text { squirrels, and } \\
\text { prairie dogs) }\end{array}$ & $\begin{array}{l}\text { birds, } \\
\text { reptiles, } \\
\text { insects }\end{array}$ \\
\hline $\begin{array}{l}\text { Swainson's } \\
\text { Hawk } 7 \\
\text { G5/S3B }\end{array}$ & $\begin{array}{l}\text { Grasslands, } \\
\text { sparse } \\
\text { shrublands, and } \\
\text { small open } \\
\text { woodlands, }\end{array}$ & $\begin{array}{l}\text { Nest in deciduous } \\
\text { trees (about } 50 \% \\
\text { of nests reused). }\end{array}$ & $\begin{array}{l}\text { Provide open grasslands } \\
\text { with occasional trees for } \\
\text { nesting and perching. }\end{array}$ & $\begin{array}{l}\text { Species unique } \\
\text { in switching from } \\
\text { primarily small } \\
\text { mammal diet to } \\
\text { insects after } \\
\text { young birds } \\
\text { fledge. }\end{array}$ & $\begin{array}{l}\text { Mid-March } \\
\text { Early } \\
\text { November }\end{array}$ & $\begin{array}{l}\text { Early May } \\
\text { to Mid- } \\
\text { July }\end{array}$ & $\begin{array}{l}\text { 28-35 days } \\
30 \text { days }\end{array}$ & 1 & $\begin{array}{l}\text { mainly } \\
\text { vertebrates } \\
\text { during } \\
\text { breeding } \\
\text { season } \\
\text { (mammals, } \\
\text { birds, \& } \\
\text { reptiles) }\end{array}$ & $\begin{array}{l}\text { invertebrates } \\
\text { at other } \\
\text { times }\end{array}$ \\
\hline \multicolumn{11}{|c|}{ Intermediate to Tall Grassland Cover Associated Species } \\
\hline $\begin{array}{l}\text { Grasshopper } \\
\text { Sparrow }{ }^{8} \\
\text { G5/S3B }\end{array}$ & $\begin{array}{l}\text { Prefers } \\
\text { moderately open } \\
\text { grasslands with } \\
\text { patchy bare } \\
\text { ground. } \\
\text { Generally avoids } \\
\text { areas with } \\
\text { extensive shrub } \\
\text { cover, although } \\
\text { some shrub } \\
\text { cover is } \\
\text { desirable. }\end{array}$ & $\begin{array}{l}\text { Ground nester: } \\
\text { nest often domed } \\
\text { with grasses and } \\
\text { side entrance. }\end{array}$ & $\begin{array}{l}\text { Provide/protect large areas } \\
\text { of contiguous grassland of } \\
\text { intermediate height with } \\
\text { moderately deep litter } \\
\text { cover and low shrub } \\
\text { density. }\end{array}$ & $\begin{array}{l}\text { May be locally } \\
\text { abundant, } \\
\text { generally rare } \\
\text { throughout } \\
\text { range. } \\
\text { Somewhat area } \\
\text { sensitive. } \\
\text { Moderate-to- } \\
\text { high nest } \\
\text { predation. }\end{array}$ & $\begin{array}{l}\text { Early May } \\
\\
\text { Early } \\
\text { September }\end{array}$ & $\begin{array}{l}\text { Mid-June } \\
\text { to Late } \\
\text { July }\end{array}$ & 8-9 days & $\begin{array}{c}2 \\
\text { (poss. 3) }\end{array}$ & insects & $\begin{array}{l}\text { rarely } \\
\text { seeds/plant } \\
\text { material in } \\
\text { summer }\end{array}$ \\
\hline $\begin{array}{l}\text { Baird's Sparrow }{ }^{9} \\
\text { prairie endemic } \\
\text { G4/S2B }\end{array}$ & $\begin{array}{l}\text { Ungrazed to } \\
\text { moderately } \\
\text { grazed, } \\
\text { generally with } \\
\text { litter depth of } \sim 2 \\
\mathrm{~cm} \text {. }\end{array}$ & $\begin{array}{l}\text { Ground nester; } \\
\text { shallow scrape at } \\
\text { base of grass } \\
\text { clump. }\end{array}$ & $\begin{array}{l}\text { Provide areas of native } \\
\text { grassland (or idle tame } \\
\text { pastures) with moderately } \\
\text { deep litter. Avoid } \\
\text { excessive grazing and limit } \\
\text { shrub encroachment. }\end{array}$ & $\begin{array}{l}\text { Scattered low } \\
\text { shrubs and } \\
\text { residual } \\
\text { vegetation from } \\
\text { last year are } \\
\text { preferred } \\
\text { habitat; area } \\
\text { sensitive (favors } \\
\text { large parcels). } \\
\text { Nesting } \\
\text { densities change } \\
\text { according to } \\
\text { local habitat } \\
\text { conditions. }\end{array}$ & $\begin{array}{l}\text { Mid-May } \\
\\
\text { Early } \\
\text { September }\end{array}$ & $\begin{array}{l}\text { Late May } \\
\text { to Late } \\
\text { July }\end{array}$ & 11-12 days & 1 & insects & $\begin{array}{l}\text { seeds/plant } \\
\text { material }\end{array}$ \\
\hline
\end{tabular}




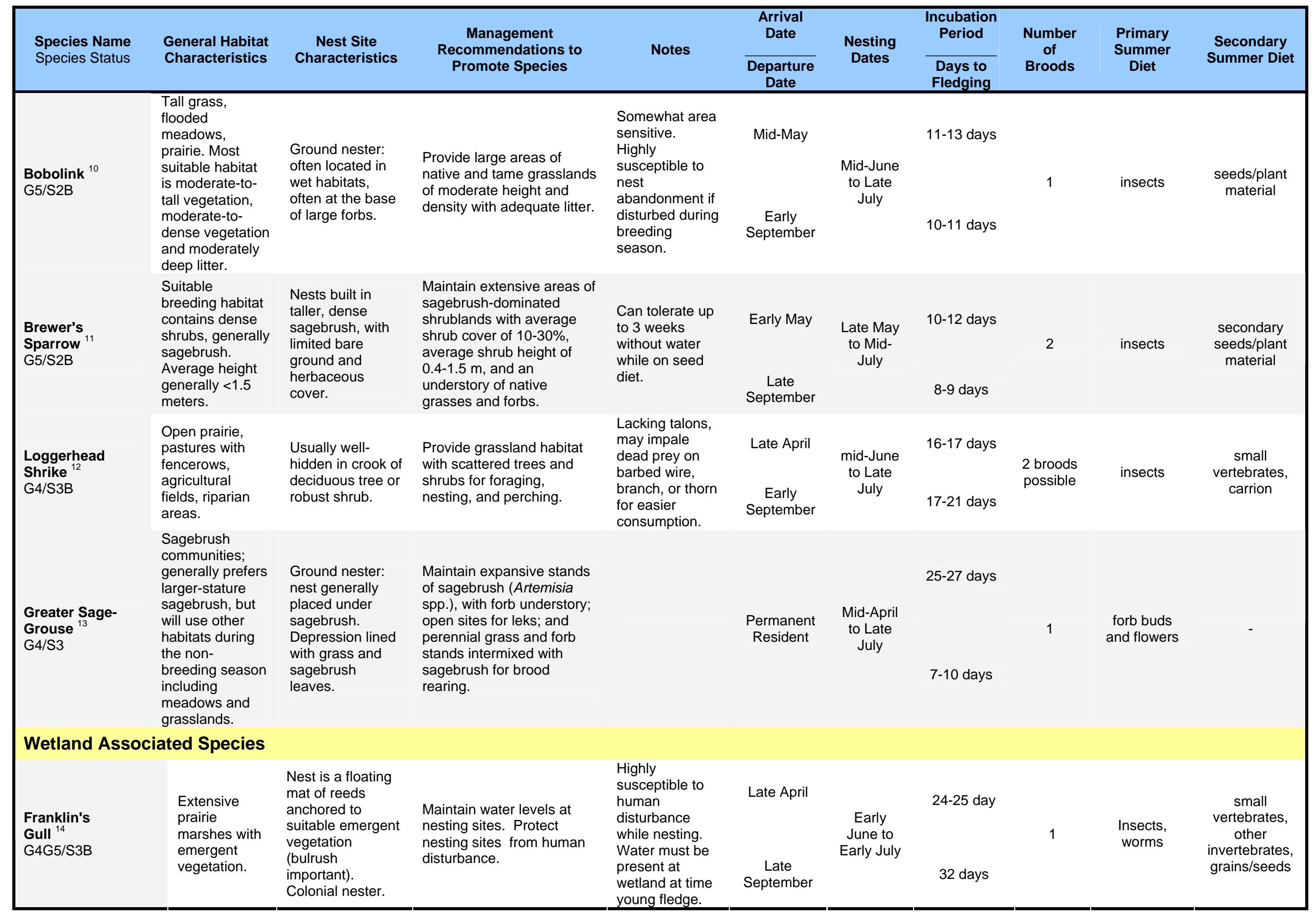




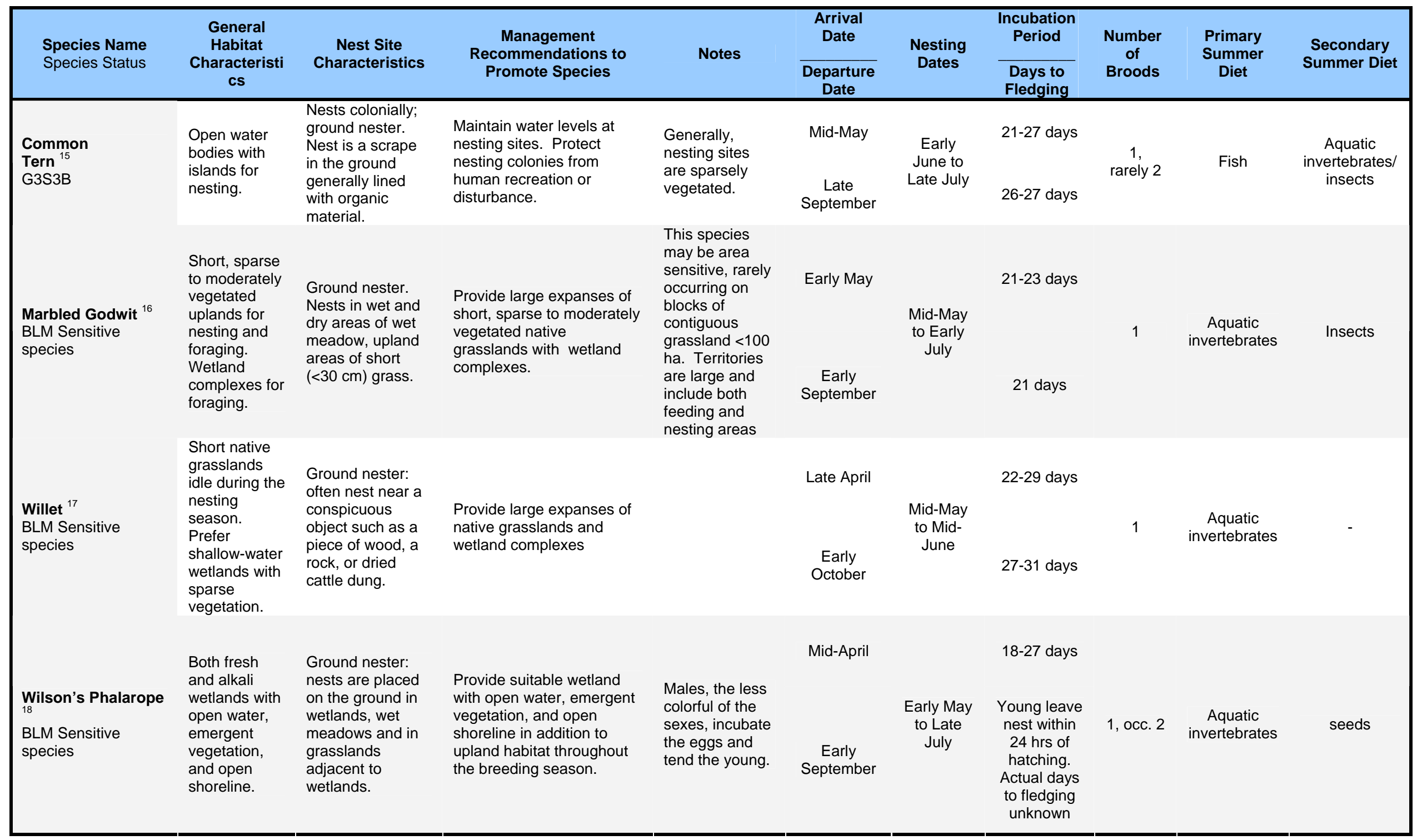

1 - With 1994, Dechant et al. 2003j

2 - Hill and Gould 1997, Dechant et al. 2003c

3 - Shane 2000, Dechant et al. 2003m

4 - Dugger and Dugger 2002, Dechant et al. 2003i

5 - Robbins and Dale 1999, Dechant et al. 2003g

6 - Bechard and Schmutz 1995, Dechant et al. 20031
7 - England et al. 1997, Dechant et al. 2003a

8 - Vickery 1996, Dechant et al. 2003d

9 - Johnson \& Igl 2001, Green et al. 2002, Dechant et al. 2003b

10 - Martin and Gavin 1995, Dechant et al. 2003k

11 - Rotenberry et al. 1999, Ehrlich et al. 1988

12 - Yosef 1996, Dechant et al. 2003e
13 - Rowland 2004, Ehrlich et al. 1988

14 - Burger and Gochfeld 1994, Ehrlich et al. 1988

15 - Nisbet 2002, Ehrlich et al. 1988

16 - Dechant et al. 2003f, Ehrlich et al. 1988

17 - Lowther et al. 2001, Dechant et al. 2003h

18 - Colwell and Jehl 1994, Ehrlich et al. 1988 